\title{
SALES-ADVERTISING RELATIONSHIP: AN APPLICATION OF PANEL DATA FROM THE GERMAN AUTOMOBILE IN- DUSTRY
}

\author{
Petr MARIEL, Cristina LÓPEZ, Karmele FERNÁNDEZ*
}

\begin{abstract}
This paper uses panel data from the German car industry for the estimation of parameters of a demand equation applying different statistical methodologies and paying special attention to advertising variables. Two important conclusions can be drawn. First, advertising plays an important role in this market but its effectiveness depends on its form and type of message; and second, the marketing policy of a firm has to take into account the size of its cars.
\end{abstract}

Key words: principal components, classification, advertising

JEL Classification: C23, M37

\section{Introduction}

This paper tries to explain the behaviour of the oligopolistic German car market using panel data from the period 1993 - 1995 applying different statistical methodologies. Our primary goal is to estimate a demand function faced by the individual car producer including the effect of advertising. Many analyses state that advertising has a limited capacity to stimulate total market growth and cast doubt on its effect in more objective tasks. We therefore seek to determine the role of advertising in this specific car market using Principal Component Analysis (PCA), Classification Analysis and Instrumental Variable Method applied to a panel data model.

We also try to relate our conclusions to existing literature. Murfin (1984) applies the Almost Ideal Demand System (AIDS) of Deaton, Muellbauer (1980) to the modelling of market share in the UK passenger car market. Most estimated effects in his study on aggregate demand are shown to conceal considerable differences between submarkets. This leads to a suggestion that the model could be improved by taking into account the existence of market segmentation and heterogeneity of car characteristics and by defining the more relevant price variables. Our conclusi-

*) Departamento de Econometría y Estadística, Universidad del País Vasco, Lehendakari Aguirre 83, E48015 BILBAO, Spain, e-mail: etpmaxxp@bs.ehu.es.

${ }^{* *}$ ) Financial aid from Gobierno Vasco (PI-1999-46) and from UPV-EHU (UPV038.321-G55/98, UPV $00038.321-13503 / 2001$ and UPV $00038.321-13631 / 2001)$ is gratefully acknowledged. 
ons support these results, and show big differences between submarkets for big, medium and small cars. We also correct the offcial list prices for quality characteristics by constructing hedonic prices. In a similar paper (see Pelsmacker, 1988) there are used data from the Belgian car market in order to estimate a modified AIDS model. Pelsmacker also shows that segmentation of the car market and a more accurate definition of the price variable improve the significance of the estimated coefficients. Moreover, he does not find marketing variables particularly relevant except for a certain extent in one of four segments analyzed.

The paper is organized as follows. Section 2 presents data and some preliminary analysis needed for a correct estimation of the model using panel data. One of these analyses presented in section 3 is a classification needed to define car classes and direct rivals for each class model. Section 4 discusses different estimation procedures applied to our data and section 5 concludes.

\section{Data, Variables and Related Issues}

In order to estimate our empirical model we use the data from the seven producers with the largest market shares in the German automobile industry: BMW $(6.9 \%)$, Mercedes (9.7\%), Opel (10.1\%), Volkswagen (23.6\%), Fiat (4.9\%), Ford $(7 \%)$ and Renault $(5.7 \%)^{1)}$. Given that for most car models (e.g. GOLF, ASTRA) and submodels (e.g. GOLF GTI, GT, TDI) the advertising variable has a zero value we formed three segments for each producer, which can be called "big", "medium" and "small" cars, to get more homogeneous data. This way we form 18 groups of the biggest-selling types of cars in Germany. The data run from January 1993 to June 1995. Sales series are the number of cars sold each month, obtained from the "Report of New Car Registration in Germany"2), which is a detailed report by car models and manufacturers.

The advertising expenditure data ${ }^{3)}$ are the observed number of pages (newspapers, journals) minutes (TV, radio) and posters devoted to the advertising of each car model under study, multiplied by the official (unknown to us) price of one page, minute or poster. Therefore, all the series are valued in German marks and represent the cost of the advertising efforts of the competitors. In order to represent the diminishing returns to scale, it is assumed that the advertising levels are the square roots of the observed advertising efforts. This reduction could be because additional units of advertising do not reach the same number of potential buyers as the first units did, or simply because the additional units are assigned to less efficient resources (see Jorgensen, 1982, Erickson, 1992).

Prices are taken from the official publication Schwacke Liste, which contains prices for every car model. Price variables for each car group are built up as a weighted index where the weight is formed using the number of cars sold and finally deflating those prices by the consumer price index ${ }^{4}$. Our original source for the price variable (Schwacke Liste) contains data on cars made by German producers. In order not to lose the remaining car models in our analysis we use the data published in the magazine Automóvil published in Spain, which provides data on all the car models in our database. These new prices are treated as former prices after

1) Data corresponding to January 1993.

2) Source: Zulassungen von fabrikneuen Personenkraftwagen in Deutschland nach Herstellern und Typgruppen, published by Kraftfahrt-Bundesamt.

3) Source: Nielsen Werbeforschung $\mathrm{S}+\mathrm{P} \mathrm{GmbH}$, Hamburg

4) Source: CD-ROM International Financial Statistics, International Monetary Fund. 
conversion from the Spanish peseta to German mark using the exchange rate of the month in which the price variable data were collected ${ }^{5}$. Before using these new data we compare prices from the two sources to ensure that the Spanish source contains the same information.

Sales series show very high seasonality (see Figure 1). One possibility is to treat this seasonality as deterministic and include a dummy variable for each month in the demand equation. But this is a very rigid solution which leads to a significant loss of degrees of freedom. The sales series available in our database are from January 1990 to June 1995. This higher number of observations (66) leads us to treat this seasonality as stochastic. We apply the X-11 (see Hilleberg, 1992) method in order to subtract the stochastic seasonal component ${ }^{6)}$ from the series, and thus obtain deseasonalyzed series, which are used in our analysis (see Figure 1). Using the magazine Automóvil we also collect the following main characteristics of each car model, which are used to form the quality adjusted price: $\mathrm{cm}^{3}(\mathrm{CM})$, horsepower (PW), length (LENG), width (WIDTH), height (HEIGHT), trunk capacity (CAPT), weight (WEIG), average fuel consumption (CONS), top speed (SPEED) and acceleration $(A C C E L)^{7)}$.

Figure 1

Sales and Seasonally Adjusted Sales for Medium Opel and BMW

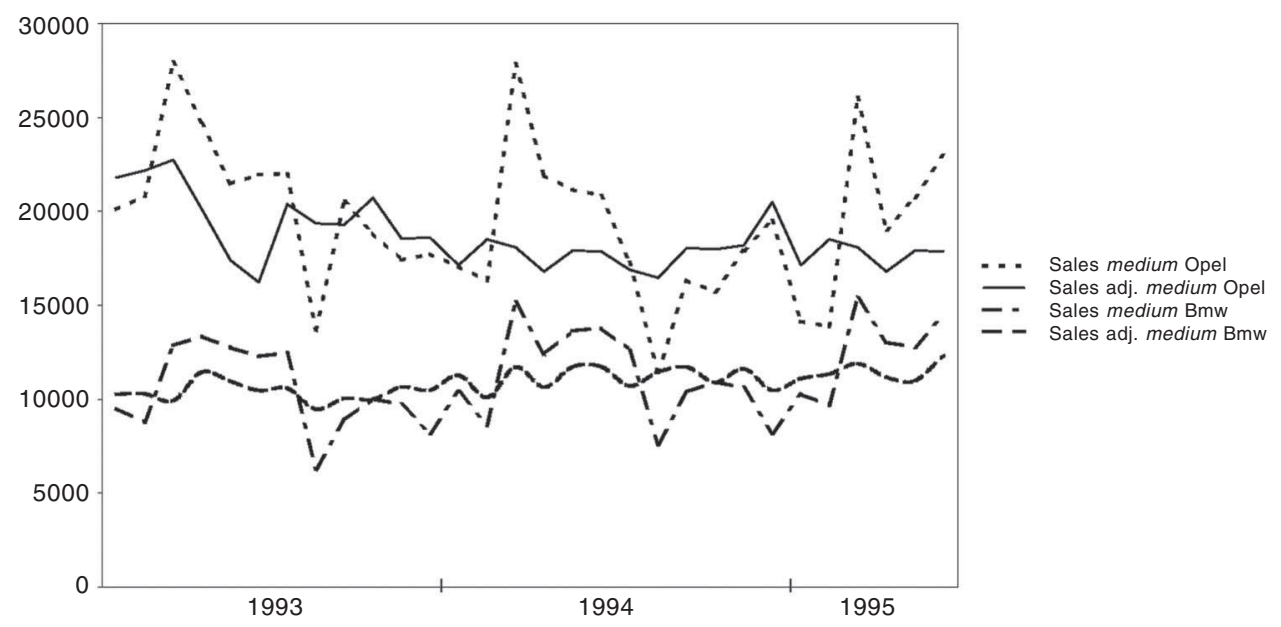

Source: Own elaboration based on data from Kraftfahrt-Bundesamt.

One of the major difficulties when analyzing the car market is the definition of a relevant car price. This is a problem mainly because official prices take no account of quality differences between models (see Griliches, 1971) and the more readily available list prices may differ from actual transaction prices (see Crafton, Hoffer, 1981). Although a different price may be established at each car retail outlet, in our case, price variable observations come directly from the producer.

5) www.xe.com

6) The seasonal component is subtracted by SPSS version 10.0.

7) acceleration from 0 to $100 \mathrm{~km} / \mathrm{h}$ in seconds 
A look at the original price variable reveals a lack of significant changes over time. The quality adjustment of price changes can be more explicitly obtained by the construction of a hedonic or quality-adjusted price definition. One of the standard procedures for correcting prices for quality is to estimate the relationship between a product's price and its quality characteristics, which in our case may be car dimension, car capacity, horsepower, etc., by means of least squares regression analysis and to define regression residuals as a measure of quality-adjusted price.

In our analysis we estimate a hedonic price function including the following quality regressors for each car: $\mathrm{cm}^{3}$, horsepower, length, width, height, trunk capacity, weight, average fuel consumption, top speed and acceleration. A competitive market with a continuum of characteristics would ensure that the hedonic price function is linear (see Arguea, Hsiao, 1993) and is defined by:

$$
P i=\delta_{0}+\delta_{1} \text { Char }_{1 i}+\delta_{2} \text { Char }_{2 i}+\ldots+\delta_{p} \text { Char }_{p i}+v_{i} \quad i=1,2, \ldots, n
$$

where Char $_{j i}$ is the jth characteristic of the ith group of cars (in our case $p=10$ and $n=18$ ) and $v_{i}$ the error term or residual used as the regressor in the estimation of car market demand.

Since we use a linear function, the coefficients of each characteristics are the estimated shadow prices (see Arguea, Hsiao, 1993). Nevertheless, many of the coefficients are not statistically significant because many of the regressors seem to measure the same attributes and they are highly collinear (see Table 1). Hence, in practice, there is strong linear dependency and the problem of selecting a set of variables, which are not highly correlated, is an empirical one.

A common procedure for reducing the number of variables in statistical exploratory studies is PCA (see Anderson, 1984). We therefore carry out a PCA for each month (from January 1993 to June 1995), obtaining 30 analyses. We take the results of the first month of our data, and compare them to the results obtained using data from other months in order to analyze possible technology variations.

We actually find no significant differences among these 30 PCA's, which is an expected result given the relatively stable development of our data over time. As shown by Arguea, Hsiao (1993), the technical specification of the products would show time trends if there were any technology variations.

Table 1

\section{Correlation Matrix}

\begin{tabular}{|l|c|c|c|c|c|c|c|c|c|c|}
\hline & CM & PW & LENG & WIDTH & HEIGHT & CAPT & WEIG & CONS & SPEED & ACCEL \\
\hline CM & 1.00 & & & & & & & & & \\
PW & 0.93 & 1.00 & & & & & & & & \\
LENG & 0.93 & 0.83 & 1.00 & & & & & & & \\
WIDTH & 0.88 & 0.80 & 0.87 & 1.00 & & & & & & \\
HEIGHT & 0.39 & 0.28 & 0.44 & 0.51 & 1.00 & & & & & \\
CAPT & 0.70 & 0.56 & 0.89 & 0.74 & 0.52 & 1.00 & & & & \\
WEIG & 0.93 & 0.92 & 0.92 & 0.87 & 0.47 & 0.77 & 1.00 & & & \\
CONS & 0.94 & 0.92 & 0.91 & 0.89 & 0.42 & 0.72 & 0.94 & 1.00 & & \\
SPEED & 0.89 & 0.94 & 0.85 & 0.87 & 0.40 & 0.65 & 0.92 & 0.90 & 1.00 & \\
ACCEL & -0.74 & -0.79 & -0.70 & -0.84 & -0.35 & -0.53 & -0.75 & -0.73 & -0.90 & 1.00 \\
\hline
\end{tabular}

Source: Own elaboration based on data from the magazine Automóvil.

The goal of the principal component method is to find the linear combinations with large variance formed by the original variables. In many exploratory studies a way of reducing the number of variables to be treated is to discard the linear combi- 
nations which have small variances and study only those with large variances. An important point is that principal components may be inputs to a multiple regression or cluster analysis (see Johnson, Wichern, 1988) which is a technique we apply in our paper. Although all principal components are required to reproduce the total system variability a number of $k$ principal components usually replace the initial $p$ variables $(k<p)$ in empirical applications.

Principal components are particular linear combinations of the $p$ observed variables $X_{1}, X_{2}, \ldots, X_{p}$ (car characteristics, so that $p=10$ ) which are observed for $n$ individuals, in our case for $n=18$ groups of cars. The observations of these variables can be placed in a matrix $(n \times p$ denoted $\boldsymbol{X}$, the elements of which are denoted $x_{i j}$ ). The linear combinations sought represent the selection of a new coordinate system obtained by rotating the original system of variables. The new axes represent directions with maximum variability and provide a simpler and more parsimonious description of the covariance structure of the observed variables.

Table 2 presents obtained eigenvalues, percentages and cumulative percentages of projected variances. The stopping rule (that is how many components will be retained and used in the posterior procedures) is subjective, since the number of components retained is based on an arbitrarily determined criterion for the amount of variation accounted for (see Dillon, Goldstein, 1984). But when factoring a correlation matrix, statistical procedures no longer hold and the retained variance criterion lacks clear meaning. Perhaps the most frequently used extraction approach is the "root greater than one" criterion originally suggested by Kaiser (1958) which proposes retaining those components whose eigenvalues are greater than one.

Table 2

Eigenvalues and Percentages of Projected Variances

\begin{tabular}{|c|c|c|c|}
\hline Number & Eigenvalue & Percentage & Cumulative percentage \\
\hline 1 & 7.9005 & 79.00 & 79.00 \\
2 & 0.9264 & 9.27 & 88.27 \\
3 & 0.5370 & 5.37 & 93.64 \\
4 & 0.3274 & 3.27 & 96.91 \\
5 & 0.1425 & 1.42 & 98.33 \\
6 & 0.0747 & 0.75 & 99.08 \\
7 & 0.0413 & 0.42 & 99.50 \\
8 & 0.0258 & 0.25 & 99.75 \\
9 & 0.0135 & 0.14 & 99.89 \\
10 & 0.0111 & 0.11 & 100.00 \\
\hline
\end{tabular}

Table 3

Coordinates and Variable-Factor Correlations ( 1 to 5 axes)

\begin{tabular}{|l|c|c|c|c|c|}
\hline \multirow{2}{*}{ Variables } & \multicolumn{5}{|c|}{ Coordinates, Var.-Fact. Correl. } \\
\cline { 2 - 6 } & 1 & 2 & 3 & 4 & 5 \\
\hline CM & -0.95 & 0.11 & -0.09 & 0.16 & 0.10 \\
PW & -0.92 & 0.31 & 0.06 & 0.20 & -0.09 \\
LENG & -0.95 & -0.07 & -0.26 & -0.04 & 0.01 \\
WIDTH & -0.94 & -0.05 & 0.09 & -0.15 & 0.26 \\
HEIGHT & -0.51 & -0.78 & 0.34 & 0.17 & -0.02 \\
CAPT & -0.80 & -0.37 & -0.39 & -0.25 & -0.10 \\
WEIG & -0.97 & 0.02 & -0.07 & 0.13 & -0.10 \\
CONS & -0.96 & 0.07 & -0.08 & 0.15 & 0.10 \\
SPEED & -0.95 & 0.17 & 0.17 & -0.03 & -0.15 \\
ACCEL & 0.84 & -0.19 & -0.38 & 0.33 & 0.04 \\
\hline
\end{tabular}


In our case, the first two factors (see Table 2) account for $88.27 \%$ of the total variance (the first factor $79 \%$ and the second $9.26 \%$ ) so, at first glance, it seems sufficient to take into account the first two factors in order to find a small number of variables which represent a high percentage of the information obtained in all variables. The variance corresponding to the remaining axes may be mainly random noise (see Lebart, Morineau, Warwick, 1984).

If the original variables have been previously standardized and we provide each individual with a mass (weight) $p_{i}$ which is usually defined by $1 / n$ the quadratic distance of each variable $j$ to the origin coincides with its variance:

$$
D^{2}(j, 0)=\sum_{i=1}^{n} p_{i} x_{i j}^{2}=1
$$

That means that the variables are placed on the unit sphere. On the other hand, the chord distance between two variables $j$ and $k$

$$
D^{2}(j, k)=\sum_{i=1}^{n} p_{i}\left(x_{i j}-x_{i k}\right)^{2}=\sum_{i=1}^{n} p_{i} x_{i j}^{2}+\sum_{i=1}^{n} p_{i} x_{i k}^{2}-2 \sum_{i=1}^{n} p_{i} x_{i j} x_{i k}
$$

The first two terms on the right hand side are equal to one and the third term is the correlation coefficient between variables $j$ and $k$ multiplied by two. Thus, the distance between two points can be expressed by the correlation coefficient $c_{j k}$ :

$$
D^{2}(j, k)=2\left(1-c_{j k}\right)
$$

The correlation coefficient is the cosine of the angle that the two variables form and the value of $D^{2}(j, k)$ reaches its minimum and maximum in the case of two collinear variables, that is $c_{j k}=-1$ and $c_{j k}=1$, respectively. Two orthogonal variables $\left(c_{j k}=0\right)$ are placed in an intermediate position. Thus if variables are properly represented on the main plane formed by the first two components, their proximity can be interpreted in terms of correlations.

The next step is to identify the first two factors obtained by the Principal Component Analysis with a small number of variables that are able to collect the maximum variance or information that those factors contain. The choice of variables must be made in such a way that those variables are not highly collinear one with another, but collect most of the information contained in the first two factors. The variablefactor correlations shown in Table 3 indicate the degree of relation between the factor and the variable. Almost all the variables are highly correlated with the first factor. The only exception is HEIGHT, which is mainly correlated with the second factor.

Figure 2 shows that two components suffice properly to represent all variables because their projections are near to the circle of unity radius and that is why the positions of the characteristics on the plane reliably indicate the correlations between the variables. This figure offers complementary information to Table 3 .

Taking into account Table 3 and Figure 2 we choose PW and LENG for their high, negative correlations with the first factor (at the same time PW has the highest positive deviation from line of factor 1 in Figure 2) and ACCEL for its positive correlation with the first factor. The last variable selected is HEIGHT for its high correlation with the second factor (and its position almost orthogonal to the remaining characteristic projections). So, the hedonic price equation is defined as follows:

$$
P i=\delta_{0}+\delta_{1} P W_{i}+\delta_{2} H E I G H T_{i}+\delta_{3} A_{C C E L_{i}}+\delta_{4} \text { LENG }_{i}+v_{i} \quad i=1,2, \ldots, 18 ;
$$

where, after an OLS estimation, the residuals $\hat{v}_{i}$ are used as the hedonic price variable. 
Figure 2

Quality Characteristics of the 18 Groups of Cars on the Main Plane

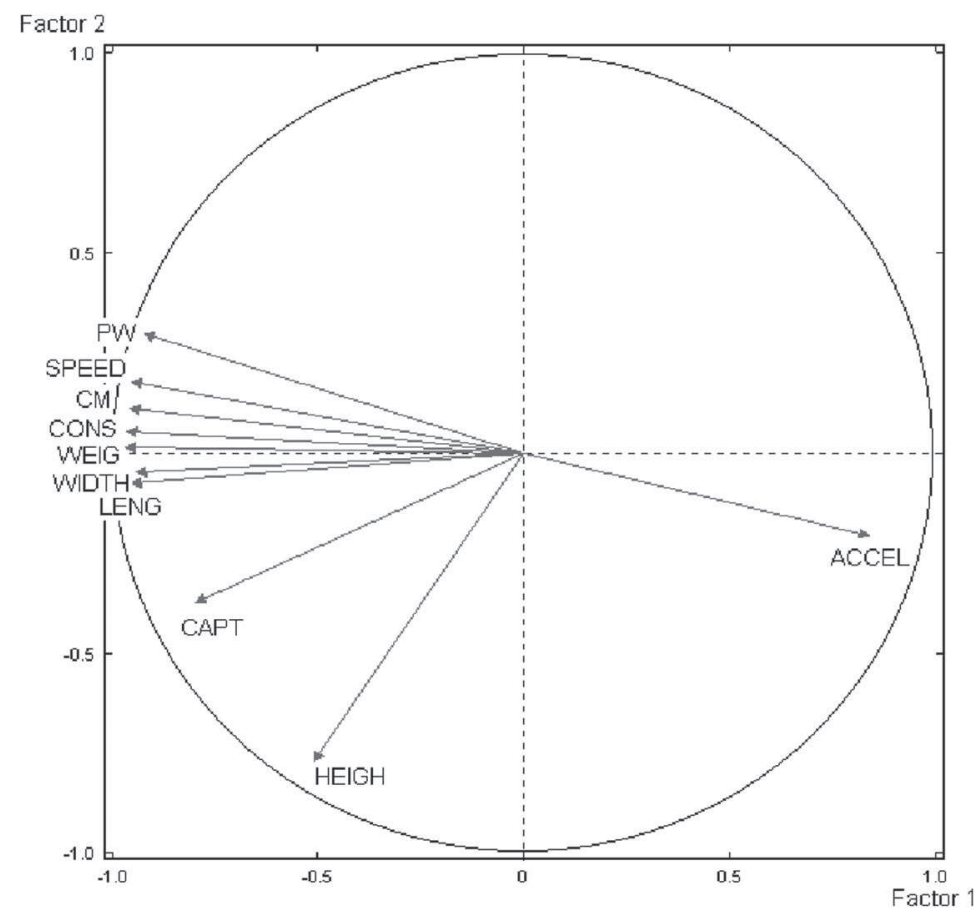

Source: Own elaboration based on data from the magazine Automóvil.

Each of the 18 groups usually brought together submodels (e.g. GTI, GT) of one model (e.g. GOLF) and we used a simple denomination of big, medium and small which does not necessarily mean that "big" of one producer is a direct competitor of a "big" of another. In order to identify the direct competitors of each group of cars we apply the following classification analysis. The results of this classification will be used in our demand equation because not only own price and advertising but also rivals' price and advertising will be used as explanatory variables.

\section{Classification}

Starting from the PCA performed above we carry out a classification analysis, using the variance criterion and following Ward's method. The variance method is based on Huygens's decomposition (see Lebart et al., 1984), where the total inertia of a set of points is equal to the sum of the intra inertia (inertia within clusters) and the inter inertia (inertia between clusters). The higher the ratio of inter inertia to total inertia is, the more homogeneous the groups of rivals will be. First, we describe the general procedure of this method applied to the original data $\boldsymbol{X}$ and subsequently apply it to the scores of the first principal components.

We consider $n$ individuals (18 groups of cars) which we have to classify as an individual set of points in a space of $p$ dimensions (10 characteristics or variables). Each point $x_{i}$ of that set of points is a vector of 10 coordinates (ith row of $\boldsymbol{X}$ ) and has a mass $m_{i}$ equal to one. The total mass of the set of points is 


$$
m=\sum_{i=1}^{n} m_{i}
$$

The square of the Euclidean distance between the points $x_{i}$ and $x_{k}$ would be denoted as:

$$
\left\|x_{i}-x_{k}\right\|^{2}=d^{2}\left(x_{i}, x_{k}\right)
$$

The inertia of the set of points is defined as the quantity:

$$
I_{\text {Total }}=\sum_{i=1}^{n} m_{i}\left\|x_{i}-g\right\|^{2},
$$

where $g$ is the overall center of gravity, that is $g=(1 / m) \sum_{i=1}^{n} m_{i} X_{i}$.

Huygens's decomposition (see Lebart et al., 1984) displays an additive decomposition of the quantity I into within-cluster and between cluster inertia as shown in the following formula:

$$
I_{\text {Total }}=\sum_{q} m_{q}\left\|g_{q}-g\right\|^{2}+\sum_{q} \sum_{i \in q} m_{i}\left\|X_{i}-g_{q}\right\|^{2}
$$

where $m_{i}$ is the mass of each vector $x_{i}, m_{q}$ is the mass of the $q$ th class, that is $m_{q}=\sum_{i \in q} m_{i}$. Finally, $g_{q}$ is the center of gravity of each subgroup created, that is $g_{q}=1 / m_{q} \sum_{i \in q} m_{i} x_{i}$. Each subgroup is treated in the next stage as a new individual.

The criterion of aggregation based on variance consists of finding at each stage one partition that minimizes the internal inertia (inertia within cluster) of each class, and at the same time maximizes the inertia between clusters. The two values included in this criteria are defined as follows:

$$
\begin{aligned}
& \text { Interia within clusters: } I_{\text {within }}=\sum_{q} \sum_{i \in q} m_{i}\left\|x_{i}-g_{q}\right\|^{2} \\
& \text { Interia between clusters: } I_{\text {between }}=\sum_{q} m_{q}\left\|g_{q}-g\right\|^{2}
\end{aligned}
$$

The ratio between inter inertia and total inertia indicates the degree of homogeneity of the classes obtained. The closer to 1 the ratio $I_{\text {between }} / I_{\text {Total }}$ is, the more homogeneous classes are formed.

Sometimes it is more efficient to obtain a classification using a limited number of factors and that is why we take into account only the first three principal components, which account for $93.64 \%$ of the total inertia explained for our final classification. In this case $x_{i}$ is formed by the first three scores $z_{i}$ which contain the principal component. Starting from the hierarchical tree shown below, an adequate number of classes should be decided, so that these classes are as homogeneous as possible with regard to the model of cars included in them. At the same time they must be as different from one other as possible. The dendogram (3) shows five quite homogeneous and intuitive classes obtained by the procedure described above.

- Class 1: Big BMW, Mercedes and Ford and Medium BMW.

- Class 2: Big Renault, Opel and Volkswagen.

- Class 3: Medium Mercedes and Ford.

- Class 4: Medium Renault, Fiat, Opel and Volkswagen.

- Class 5: Small Opel, Volkswagen, Ford, Renault and Fiat. 
Figure 3

\section{Dendrogram}

\begin{tabular}{cl}
1 & Big BMW \\
2 & Big Mercedes \\
3 & Medium BMw \\
4 & Big Ford \\
5 & Big Renault \\
6 & Big Opel \\
7 & Big Volkswagen \\
8 & Medium Mercedes \\
9 & Medium Ford \\
& \\
\hline 10 & Medium Renault \\
11 & Medium Fiat \\
12 & Medium Opel \\
13 & Medium Volkswagen \\
16 & Small Opel \\
17 & Small Volkswagen \\
16 & Small Fiat \\
\hline 14 & \\
\hline 15
\end{tabular}

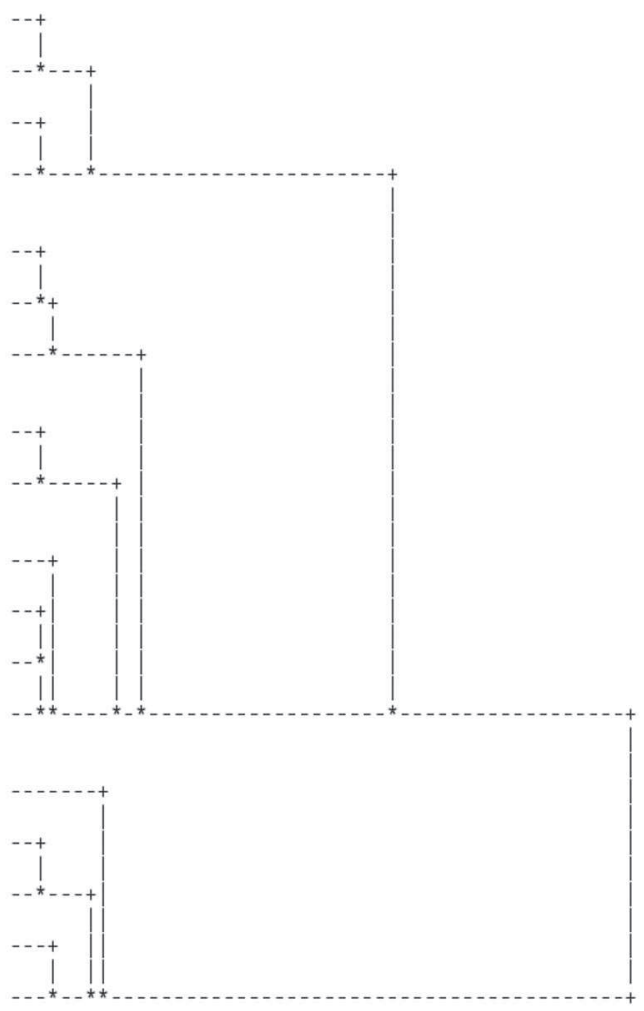

The link between classification and PCA is made by positioning the centres of the car groups on the factorial plane (see Figure 4) of the individuals formed by the first two principal components ${ }^{8)}$.

8) For PCA and classification we have used SPAD software package, version 5.0. 
Figure 4

Class Projections on the Factorial Plane

Factor 2

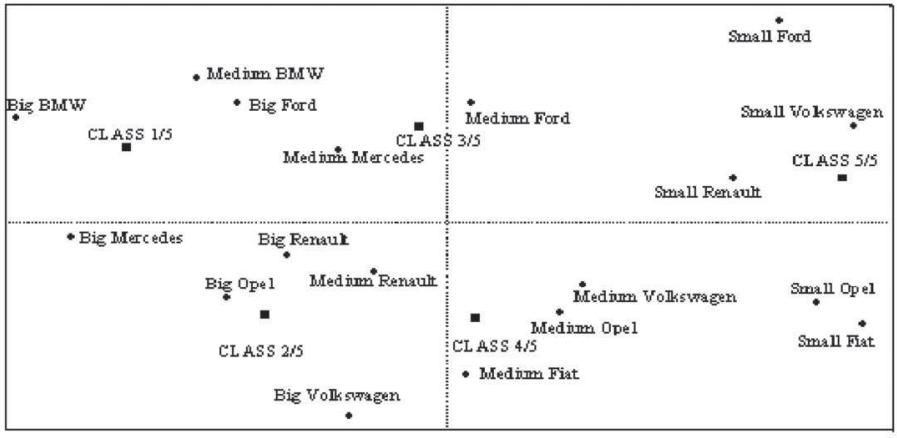

Factor

Source: Own elaboration based on data from the magazine Automóvil.

\section{Estimation Results}

After the computation of the hedonic prices and identification of the direct rivals of each group of car by application of PCA and classification analysis, we can estimate coefficients of the demand equation using a panel data model. We consider an oligopolistic market made up of 18 differentiated groups of cars whose sales, price and advertising variables are observed over 30 months from January 1993 to June 1995. Sales of group $i$ in time period $t$ are denoted by sales $s_{i t}$, advertising level in newspapers and journals and on TV and radio by adsjourn $n_{i t}$ and adsrtv atespecti- $_{\text {it }}$ vely, hedonic price by pricehed ${ }_{i t}$, and finally radsjourn ${ }_{i t}$, radsrtvit and rpricehed $_{i t}$ represent the advertising level and hedonic price established by the direct rivals which have been fixed previously by the classification procedure. If there is more than one direct rival in a class, we use a sample mean of all the remaining groups in the class. We also include one exogenous macro-variable, namely the rate of interest, which

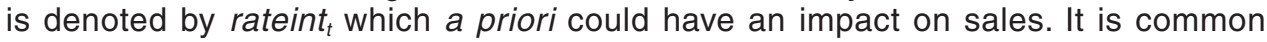
procedure in the relevant literature to include a macro-variable in this kind of models.

Based on the articles of Pelsmacker (1988) and Murfin (1984) the demand function faced by firm $i$ in period $t$ is defined as:

$$
\begin{aligned}
\text { sales }_{i t}= & \beta_{1} \text { adsrtv }_{i t}+\beta_{2} \text { adsjourn }_{i t}+\beta_{3} \text { pricehed }_{i t}+\beta_{4} \text { radsrtv }_{i t} \\
& +\beta_{5} \text { radsjourn }_{i t}+\beta_{6} \text { rpricehed }_{i t}+\beta_{7} \text { rateint }_{t}+\mu+\alpha_{i}+\varepsilon_{i t}, \\
& i=1,2, \ldots, 18, \quad t=1,2 \ldots, 30
\end{aligned}
$$

where $\mathrm{a}_{i}$ is the individual effect $\left(\sum_{i=1}^{n} \alpha_{i}=0\right)$ and $\varepsilon_{i t}$ is the error term. Model (1) allows us to analyze the effect of own and rivals' advertising and price on sales. Basic economic theory indicates that the sign of the coefficient of own price $\left(\beta_{3}\right)$ is likely to be negative and that of the rivals' price $\left(\beta_{6}\right)$ positive. The effect of advertising is, however, not so straightforward. Own advertising should have a positive effect ( $\beta_{1}$, $\left.\beta_{2}>0\right)$ and the sign of $\beta_{4}$ and $\beta_{5}$ depends on the nature of advertising. Advertising is cooperative (informative) if the effect of the rivals' advertising on own future and discounted sales is positive. But advertising is predatory if this effect is negative (see 
Friedman, 1983, and Slade, 1995). Applying this rule to our static model (1) we can say that advertising is cooperative if $\beta_{4}, \beta_{5}>0$ and predatory if $\beta_{4}, \beta_{5}<0$.

The question now is how to treat the individual effects, $\alpha_{i}$. Are they fixed or random? The fixed effects model is a reasonable approach when we can be sure that differences between units can be explained by parametric movements of the regression function. In our model, differences between units refer to differences between groups of cars. The groups of cars analyzed cover almost $70 \%$ of the market. They are clearly defined and are not interchangeable. Hence, it is reasonable to assume that we face a fixed effects model. Moreover, it also seems reasonable to assume that individual effects, $\alpha_{i}$, of each group of cars must be represented by a parametric movement rather than assuming that they are a realization of a random variable.

Table 4

Ordinary Least Square Estimation (fixed effects model)

\begin{tabular}{|l|c|c|}
\hline Parameter & Estimation & $t$ Statistic \\
\hline Adsrtv & 0.155 & $2.17^{*}$ \\
Adsjourn & -0.047 & -0.85 \\
Pricehed & -0.035 & -1.84 \\
Radsrtv & 0.070 & 0.84 \\
Radsjourn & 0.085 & 1.62 \\
Rpricehed & -0.002 & 0.07 \\
Rateint & -134.344 & -1.04 \\
Constant & 6303.173 & $3.90^{*}$ \\
\hline$F(7 ; 515)=$ & 2.250 & \\
Prob $>F=$ & 0.028 & \\
F test that all $\alpha_{i}=0: \mathrm{F}(17,515)=$ & 13.200 & \\
\hline
\end{tabular}

* Significant at $5 \%$.

Source: Own elaboration.

Assuming $\alpha_{i}$ to be fixed, equation (1) can be treated as a classical regression model (sometimes called Least Square Dummy Variable Model) and the Ordinary Least Square Estimator (presented in Table 4) has a desirable properties. The general conclusion is that the $t$ statistics are very low, which may be due to the fact that our panel is very "narrow" (only 18 groups of cars). If we pay attention to the variables with the highest $t$ statistics the following conclusions can be drawn.

Own advertising in TV and radio has a significant positive effect on sales. However, own advertising in journals and newspapers does not seem to have a major effect on sales. The effect of the own price variable is, as expected, negative and the "rivals" variable has a very low $t$-statistic, which may be a consequence of complex interactions between direct rivals in each class.

The next point to analyze is the dynamics we might face in our data. Car sales usually behave cyclically, with each cycle usually lasting years. Dynamics can be included in the model in different ways, but we consider the following two possibilities.

1. If $\varepsilon_{i t}=\gamma \varepsilon_{i t-1}+e_{i t}$ in $y_{i t}=\beta x_{i t}+\alpha_{i}+\varepsilon_{i t}$, then $y_{i t}=\beta x_{i t}+\alpha_{i}+\frac{e_{i t}}{(1-\gamma L)}$

2. If $y_{i t}=\gamma y_{i t-1}+\beta x_{i t}+\alpha_{i}+\varepsilon_{i t}$, then $y_{i t}=\frac{\beta}{(1-\gamma L)} x_{i t}+\frac{\alpha_{i}}{(1-\gamma)}+\frac{1}{(1-\gamma L)} \varepsilon_{i t}$ 
In the first specification, where dynamics is included by assuming an AR(1) process in the error term, the present values of the endogenous variable depend on the present values of the regressors and on the present and past values of the errors. In the second specification, where the dynamics is included by using endogenous lagged variables, the present values of the endogenous variable depend on the present and past values of the regressors and errors ${ }^{9}$.

Table 5 presents the estimations of the fixed effects model (1), assuming that the error term follows an AR(1) process (columns 2 and 3 ) and adding a lagged endogenous variable as explanatory (columns 4 and 5 ). In the first case, the estimation procedure is similar to the known Cochrane-Orcutt method applied to panel data. Here we obtain lower $t$ statistics than in the previous estimations. The estimation of

Table 5

Autoregressive estimation (fixed effects model)

\begin{tabular}{|l|c|c|c|c|}
\hline \multirow{2}{*}{ Parameter } & \multicolumn{2}{|c|}{ Autoregressive estimation } & \multicolumn{2}{c|}{ Instrumental Variables estimation } \\
\cline { 2 - 5 } & Estimation & $t$ Statistic & Estimation & $t$ Statistic \\
\hline Sales (one lag) & & & 0.086 & 1.13 \\
Adsrtv & 0.076 & 1.01 & 0.097 & 1.53 \\
Adsjourn & -0.027 & -0.48 & -0.022 & -0.51 \\
Pricehed & -0.026 & -1.10 & -0.044 & -1.22 \\
Radsrtv & 0.053 & 0.60 & 0.110 & 1.17 \\
Radsjourn & 0.041 & 0.78 & 0.047 & 0.65 \\
Rpricehed & -0.002 & -0.06 & -0.047 & -0.72 \\
Rateint & -117.402 & -0.63 & 0.018 & 0.00 \\
Constant & 4709.290 & $2.81^{*}$ & -18.480 & -0.29 \\
\hline$\rho(\mathrm{AR}(1))$ & 0.250 & \multicolumn{4}{|l}{} \\
\hline
\end{tabular}

* Significant at 5\%.

Source: Own elaboration.

the parameter $\rho$ of the autoregressive process that underlies the errors gives a very low point estimation of 0.25 . This indicates that dynamics is not so important in our data set as we expected at first glance. In the second case, the inclusion of a lagged endogenous variable significantly affects the properties of the classical least squared estimators. The ordinary least squares from Table 4 is no longer a consistent estimator (see Hsiao, 1991) in this kind of model. An instrumental variable method or maximum-likelihood procedure must be used to get consistent estimations. A brief review of possible estimation procedures can be found in Arellano, Bover (1990). We use the Arellano, Bond (1991) instrumental variable method ${ }^{10)}$ here, because of the refined way in which it forms the instrumental variable, (see Arellano, Bond, 1991). Columns 4 and 5 of Table 5 present the instrumental variable estimation which uses the robust estimator of the variance-covariance matrix of the estimated parameters, which takes possible heteroskedasticity into account.

9) Note that $\frac{\beta}{(1-\gamma L)} X_{i t}=\sum_{s=0}^{\infty} \gamma^{s} L^{s}\left(\beta X_{i t}\right)=\sum_{s=0}^{\infty} \beta \gamma^{s} X_{i,(t-s)}$.

10) We have used Stata (see http://www.stata.com/) version 7.0 because it includes not only usual econometric analyses but also a number of procedures found only in highly specialized software (see Kolenikov, 2001). The instrumental variables estimation procedure (see Arellano, Bond, 1991) we applied to estimate the equation (1) is directly implemented in Stata (see xtabond function in StatCorp., 2001). 
We note that the coefficient of the lagged sales and its corresponding $t$-ratio are very low, confirming our suspicion that the underlying dynamics is not high. The remaining variables are not significant at $5 \%$ either which indicate a bad fit of this dynamic model.

Finally, following the conclusions of Pelsmacker (1988), we segment the car market and carry out an analysis of each submarket (small, medium and big cars) separately. We present the final estimations based on a simple specification of a static model with fixed effects because the previous estimations do not support strong dynamic behaviour of our data.

Table 6

Estimation of the Fixed Effects Model Using Segmented Data

\begin{tabular}{|l|c|c|c|c|c|c|}
\hline \multirow{2}{*}{ Parameter } & \multicolumn{2}{|c|}{ Small cars } & \multicolumn{2}{c|}{ Medium cars } & \multicolumn{2}{c|}{ Big cars } \\
\cline { 2 - 7 } & Estimation & $t$ Statistic & Estimation & $t$ Statistic & Estimation & $t$ Statistic \\
\hline Adsrtv & -0.043 & -0.30 & 0.239 & $2.42^{*}$ & 0.187 & 1.18 \\
Adsjourn & 0.001 & 0.02 & -0.103 & -1.22 & -0.080 & -0.82 \\
Radsrtv & 0.153 & 0.54 & 0.253 & 1.88 & -0.063 & -0.53 \\
Radsjourn & 0.175 & 0.88 & -0.084 & -0.72 & 0.099 & 1.63 \\
Pricehed & -0.109 & $-2.30^{*}$ & -0.068 & $-2.97^{*}$ & 0.183 & $2.34^{*}$ \\
Rpricehed & -0.235 & -1.28 & 0.073 & $-2.00^{*}$ & 0.142 & 1.63 \\
Rateint & 138.602 & 0.41 & -509.510 & $-2.68^{*}$ & -97.837 & -0.24 \\
Constant & 3477.408 & 0.83 & 10973.860 & $4.57^{*}$ & 6891.190 & 1.32 \\
\hline
\end{tabular}

* Significant at $5 \%$.

Source: Own elaboration.

Table 6 presents the final estimations based on model (1) segmenting the market into three submarkets for small, medium and big cars according to our previous classification. As classes 2 and 3 contain few groups of cars we put together classes 2, 3 and 4 according to the dendogram (see Figure 3), obtaining three classes called small, medium and big cars. Our results confirm the importance of the segmentation of the market because the behaviour of consumers in the different markets seems to be completely different. This is a very important issue because producers would have to choose different marketing strategies in each segment if their marketing variables are to have the expected effect.

The only significant variable at $5 \%$ in the small cars market is own price. No advertising variable is significant in this segment. The consumers in the segment are usually young people looking for their first car and that is why the budget restriction is decisive for them. Big advertising campaigns in this segment will probably not have the expected reaction.

In the medium car segment we find many relevant variables: own price, rivals' price, own advertising on radio and TV, interest rate and rivals' advertising on radio and TV is significant at $10 \%$. The consumers in this segment pay attention to both price and advertising. Therefore, marketing strategies can combine new advertising messages with price changes to obtain the expected reaction. The consumers in this segment usually have higher purchasing power than small car buyers, and can therefore react to advertising by purchasing a more expensive car if its characteristics are more suitable for them. The budget restriction is not the only decisive rule for them.

The own and rivals' advertising variables in radio and TV have a positive effect. Own price has the expected negative sign and a relatively high $t$ statistic confirms 
its relative importance. We should highlight the positive coefficient of rivals' advertising on radio and TV. It is very important to indicate that advertising in the market analyzed is cooperative (informative). This may be quite a surprising result, given that the car market is very competitive, but there are a lot of advertising messages describing new technical innovations included in a new car. These innovations are usually quickly offered by all direct rivals, so the messages can have an informative effect.

The big cars market is really a very interesting segment. The only significant variable here is own price, but with a positive sign. We know that buyers of these expensive cars usually have no budget restrictions but they do not seem to pay much attention to advertising either. Their behaviour is probably based on brand loyalty and at first sight the positive sign on the price variable seems to contradict classical economic theory, but there are many papers where this unexpected behaviour is described. Owning or consuming certain goods (luxury cars) defines an individual's position in a society or in particular subgroups of a society (see Rauscher, 1992). This may result in unexpected results concerning demand.

\section{Conclusions}

We use panel data from the German car industry to estimate parameters of a demand equation, paying special attention to advertising variables. We have found out that advertising plays an important role in this market but its effectiveness depends on its form and type of message. This is one of the conclusions stated by Lambin (1976). The parameters estimated indicate that advertising in this competitive market is informative in nature. This surprising conclusion is also found in Mariel (1997) and can be explained by featuring of exhibitions of new car elements (ABS, side bars) which inform customers of rival manufactures who buy cars with similar characteristics.

The building of marketing models is not an easy task and there is a large stream of marketing literature that deals with the effect of advertising and other marketing mix on sales or market shares (see Hanssens, Parsons, Schultz, 2001; Leeflang, Wittink, Wedel, Naert, 2000). Our model tries to find out new insights in the Market Response Models (see Vakratsas, Ambler, 1999) stressing that for a rigorous analysis of the effect of advertising, different statistical methodologies should be applied and the homogeneity of the analyzed products should be carefully analyzed. In order not to waste resources and to conduct marketing campaigns adequately it is necessary to take into account more homogeneous submarkets such as those presented in Table 6 . Thereby, in our case, the marketing policy to be undertaken by a firm must take into account the size of its cars.

All this issues can help to build standardized models which should be useful for marketing management and senior executives (see Hanssens, Leeflang, Wittink, 2004).

References

Anderson, T. (1984), An introduction to Multivariate Statistical Analysis, 2nd ed., New York: John Wiley \& Sons.

Arellano, M., Bond, S. (1991),"Some Tests of Specification for Panel Data: Monte Carlo Evidence and an Application to Employment Equations." Review of Economic Studies, 58, pp. 277-297.

Arellano, M., Bover, O. (1990), "La econometría de datos de panel." Investigaciones Económicas, pp. 3-45. 
Arguea, N., Hsiao, C. (1993), "Econometric Issues of Estimating Hedonic Price Functions." Journal of Econometrics, 56, pp. 243-267.

Crafton, S., Hoffer, G. (1981), "Estimating a Transaction Price for New Automobiles." Journal of Business, 54, pp. 611-621.

Deaton, A., Muellbauer, J. (1980), "An Almost Ideal Demand System." The American Economic Review, 70, pp. 312-326.

Dillon, W., Goldstein, M. (1984), Multivariate Analysis, Methods and Applications. New York: John Wiley and Sons.

Erickson, G. M. (1992), "Empirical Analysis of Closed-loop Duopoly Advertising Strategies.” Management Science, 38, pp. 1732-1749.

Friedman, J. W. (1983), "Advertising and Oligopolistic Equilibrium." The Bell Journal of Economics, 14, pp. 464-473.

Griliches, Z. (1971), Hedonic Price Indexes Revisited. Cambridge, Massachusetts: Harvard University Press.

Hanssens, D., Leeflang, P., Wittink, D. (2004), "Market Response Models and Marketing Practice." Applied Stochastic Models in Business and Industry, forthcoming.

Hanssens, D., Parsons, L., Schultz, R. (2001), Market Response Models. Boston/Dordrecht/London: Kluwer Academic Publishers.

Hilleberg, S. (1992), "The X-11 Method in Modelling Seasonality Advanced Texts in Econometrics.", Oxford: Oxford University Press.

Hsiao, C. (1991), Analysis of Panel Data, Cambridge: Cambridge University Press.

Johnson, R., Wichern, D. (1988), Applied Multivariate Statistical Analysis, Prentice Hall.

Jorgensen, S. (1982), "A Survey of Some Differential Games in Advertising." Journal of Economic Dynamics and Control, 4, pp. 341-369.

Kaiser, H. F. (1958), "The Varimax Criterion for Analytic Rotation in Factor Analysis." Psycometrika, 23, pp. 187-200.

Kolenikov, S. (2001), "Review of STATA 7." Journal of Applied Econometrics, 16, pp. 637-646.

Lambin, J. (1976), Advertising, Competition and Market Conduct in Olipoly over Time, Amsterdam: North Holland Publishing Company.

Lebart, L., Morineau, A., Warwick, K. (1984), Multivariate Descriptive Statistical Analysis, New York: John Wiley and Sons.

Leeflang, P., Wittink, D., Wedel, M., Naert, P. (2000), Building Models for Marketing Decisions, Boston/Dordrecht/London: Kluwer Academic Publishers.

Mariel, P. (1997), "A Dynamic Model of Advertising Competition: An Empirical Analysis of Feedback Strategies." Kybernetika, 33, pp. 633-648.

Murfin, A. (1984), "Market Shares in the U.K. Passenger Car Market: Marketing, Expenditure and Price Effects, 1975-80." Applied Economics, 16, pp. 611-632.

Pelsmacker, P. (1988), "Marketing, Expenditure and Quality-Adjusted Price Effects on Market Share Evolution in a Segmented Belgian Car Market (1972-81)." Applied Economics, 20, pp. 15-30.

Rauscher, M. (1992), "Keeping up with the Joneses. Chaotic Patterns in a Status Game." Economic Letters, 40, pp. 287-290.

Slade, M. (1995), "Product Rivalry with Multiple Strategic Weapons: An Analysis of Price and Advertising Competition." Journal of Economics and Management Strategy, 4, pp. 445-476.

StatCorp. (2001), Stata Statistical Software: Release 7.0, College Station, TX: Stata Corporation.

Vakratsas, D., Ambler, T. (1999), "How Advertising Works: What Do We Really Know?" Journal of Marketing, 63, pp. 26-43. 